Originales

\title{
Para qué sirve y quién debe hacer el seguimiento después del tratamiento del paciente con cáncer
}

\author{
J. Expósito Hernández', A. Dadet²
}

\section{Resumen}

- Objetivos: estudiar las características de una consulta de seguimiento de un servicio de radioterapia de un hospital que ofrece tratamiento completo del cáncer y valorar su utilidad en términos de diagnóstico de recaídas, molestias al paciente y carga de trabajo.

- Material y métodos: hemos medido durante 52 semanas consecutivas el número de pacientes citados/atendidos por día en una consulta semanal; la localización del tumor, el tiempo tras el tratamiento, el número de servicios que le hacen seguimiento y el número de sospechas clínicas de recidiva.

- Resultados: se recogen 715 visitas de 524 pacientes distintos (el $40 \%$ una visita, $40 \%$ dos visitas y $20 \%$ más de dos). La media de citas es de 18 por día (mediana 19, moda 24) de los que asisten 16 en promedio. Dos pacientes no acuden a la visita programada y uno lo hace fuera de cita. Predominan las pacientes de mama, cabeza y cuello, endometrio, cérvix y pulmón. El $74 \%$ es seguido en dos servicios y el $10 \%$ en más de 2, con media tras la radioterapia de 2,5 años (21\% a más de cinco años). Se detectan 28 sospechas de recaída (5,4\% de pacientes) y se confirman 6 ( $21 \%$ de sospecha, 1,2\% de pacientes): 4 locales y dos a distancia.

- Comentario: el seguimiento clínico tras el tratamiento persigue objetivos tales como controlar los efectos secundarios, detectar precozmente las posible recaídas y ofrecer al enfermo una relación más continua en el tiempo. Sin embargo, por las características del manejo de estos enfermos (multidisciplinaria), el seguimiento puede hacerse de forma engorrosa, redundante e ineficaz.

Palabras clave:

Cáncer. Seguimiento. Utilidad.

Oncología, 2004; 27 (9):544-547

1 Servicio de Radioterapia

2 Unidad de Calidad.

Hospital Universitario Virgen de las Nieves (Granada) 


\section{Summary}

- Purpose: To study the characteristics of a follow-up consultation in a radiotherapy unit of a full equipped hospital, and to analyze the usefulness of this activity for early diagnosis of relapse, patients inconveniences and work overload.

- Material and methods: We measured every day during 52 consecutive weeks the number of patients appointed/assisted, their tumor location, the time after treatments, the number of different units involved in the follow-up, and the number of suspected clinical relapses.

- Results: 715 consultations of 524 patients were collected, corresponding one, two and more than two consultations to $40 \%, 40 \%$ and $20 \%$ of the patients respectively. An average of 18 patients were appointed per day (median 19, mode 24), and 16 were attended; two patients did not attend the appointments, and one did it out of appointment. Most of the tumor locations corresponded to breast, head and neck, uterus and lung cancer. $74 \%$ of the patients were followed in two different units and $10 \%$ in more than two, with an average time after radiotherapy of 2.5 years $121 \%$ more than 5 years). 28 relapses (5.4\% of the patients) were suspected and 6 were confirmed $(21 \%$ of the suspected cases, $1.2 \%$ of the patients), correponding 4 to local and 2 to distant relapses.

- Conclusions: The clinical follow-up of treated cancer patients seeks several objectives, as the control of side effects, the detection of early disease relapses, and the maintenance of a closer relationship with the patients. Nevertheless, because of the characteristics of the management and the multidisciplinary approach of cancer treatment, it may become redundant and ineffective.

Key words: Cancer. Follow-up.

\section{Introducción}

Finalizado el plan de tratamiento, los pacientes de cáncer reciben un seguimiento clínico que tiene varios objetivos. Persigue controlar los posibles efectos secundarios de la enfermedad y los derivados de los tratamientos y estar atento para el diagnóstico rápido de posibles recaídas, metástasis o segundos tumores'. Dos objetivos no menos importantes son que el enfermo se sienta atendido e incrementar la experiencia clínica mediante registros adecuados que permita conocer los resultados e introducir futuras mejoras en otros casos. Recientes revisiones han puesto el acento en la necesidad de revisar la utilidad para los pacientes de estos seguimientos y en las pruebas más eficientes a que deben ser sometidos y por cuánto tiempo ${ }^{2}$.

El hecho de que el tratamiento más recomendado sea de carácter multidisciplinario y que los enfermos sean mayoritariamente tratados en hospitales generales (no monográficos), ha puesto sobre la mesa dos cuestiones. Por una parte la falta de definición de quién ostenta la responsabilidad asistencial sobre el paciente y en función de qué (puerta de entrada del proceso, localizaciones tumorales y armas terapéuticas que cada uno conoce y en la que es experto). De otra la observación de que podríamos estar haciendo un seguimiento engorroso y redundante (en varios servi- cios), que implica un esfuerzo no justificado para el paciente (tiempo y desplazamientos) y para los servicios (repetición de consultas y pruebas complementarias) ${ }^{3}$.

El objetivo de este trabajo es describir las características de este seguimiento hecho desde una consulta de radioterapia de un hospital general $(H$. U. Virgen de las Nieves, Granada) que puede ser un ejemplo bastante significativo de la situación general, al tratarse de un hospital de tercer nivel de complejidad que tiene una oferta completa para el tratamiento del cáncer (con radioterapia incluye radiocirugía, TBI, braquiterapia). El hospital cuenta con una comisión central de tumores activa, y un razonable nivel de calidad en la gestión de estos pacientes (centralización de citas de consultas y pruebas diagnósticas, protocolos multidisciplinar, comisiones clínicas). No existe sin embargo consenso explícito de seguimiento compartido entre diferentes servicios. La experiencia puede permitir proponer estrategias que faciliten mejorar el seguimiento en este tipo de pacientes.

\section{Material y métodos}

Se trata de un estudio descriptivo a partir de los datos recogidos en una consulta semanal de seguimiento 
TABLA I

Pacientes según localizaciones tumorales y servicios que realizan seguimiento

\begin{tabular}{lccccc}
\hline Localización & No & \% & 1 Servicio* & 2 Servicios* & >2 Servicios* \\
\hline Mama & 246 & 37,8 & 5,4 & 88,8 & 5,8 \\
Cabeza y cuello & 111 & 17,1 & 8,3 & 83,5 & 8,3 \\
Cérvix & 54 & 8,3 & 9,3 & 64,8 & 25,9 \\
Endometrio & 85 & 13,1 & 21,7 & 57,8 & 20,5 \\
Pulmón & 42 & 6,5 & 39,0 & 61,0 & 13,0 \\
Próstata y vejiga & 23 & 3,5 & 4,3 & 82,6 & 19,2 \\
Recto & 26 & 4,0 & 26,9 & 53,8 & 8,5 \\
Otros & 36 & 5,4 & 42,9 & 48,6 & 3,8 \\
Cerebro & 28 & 4,3 & 38,5 & 74,7 & 9,9 \\
Total & 715 & 100 & 14,7 & 74,9 & \\
\hline
\end{tabular}

* expresado en porcentajes.

postratamiento de un servicio de radioterapia. Dicho servicio participa activamente en todas las comisiones clínicas existentes. En situaciones habituales, tras el tratamiento el enfermo acude a consulta de radioterapia programada a las $4-6$ semanas y posteriormente cada tres-cuatro meses por dos años, cada seis por tres años y luego de forma anual. La organización interna de este servicio asigna a cada paciente un especialista de staff responsable.

Recogemos de una de consulta de seguimiento durante 52 semanas consecutivas (septiembre 2002agosto 2003) las variables siguientes: número de pacientes citados y número de pacientes atendidos cada día; de cada paciente, la localización del tumor, el tiempo desde la finalización del tratamiento con radioterapia, el número de servicios que le hacen seguimiento y la detección de incidencias durante la consulta (sospechas clínicas de recidiva o metástasis) y su posterior confirmación o no, así como los pacientes a los que se plantea el alta del servicio y los que la aceptan.

Se presenta un análisis descriptivo de las variables estudiadas, mediante tablas de frecuencias y estadísticos de tendencia central y dispersión (media, mediana y moda). Todos los datos han sido analizados con el programa SPSS.

\section{Resultados}

El total de visitas realizadas en el intervalo del estudio ha sido de 715 que corresponden a 524 pacientes distintos. La media de pacientes/ día citado es de 18 (mediana 19, moda 24) y la de pacientes que asisten de 16 (mediana y moda 17). Durante las 52 semanas el $40 \%$ de los pacientes acude una vez, otro $40 \%$ lo hace dos veces y un $20 \%$ lo hace más de dos veces. En promedio dos pacientes citados faltan a su cita cada día (por olvido o confusión, $80 \%$, o nuevo tratamiento en otro servicio, $10 \%$ ). Un paciente como media acude sin estar citado cada día. Las causas son complicaciones del tratamiento (75\%) o empeoramiento sintomático $(15 \%)$.

Por localizaciones tumorales, predominan las pacientes de mama, cabeza y cuello, endometrio, cérvix y pulmón (Tabla I). El número de servicios distintos donde un paciente ha sido visto muestra que el $15 \%$ es atendido sólo en radioterapia, el $74 \%$ por dos (radioterapia y otro) y el $10 \%$ por más de 2 servicios. Cuando se agrupan por localizaciones tumorales los tumores de cérvix, endometrio y recto son los que con ma-

TABLA \|

Tiempo transcurrido desde la finalización del tratamiento e incidencias (sospecha de recaídas, propuesta de alta) en la consulta

\begin{tabular}{lcccc}
\hline Tiempo & № & $\%$ & $\begin{array}{c}\text { Sospecha } \\
\text { Recaídas (No) }\end{array}$ & $\begin{array}{c}\text { Alta } \\
\text { (No) }\end{array}$ \\
\hline$<1$ año & 299 & 42,0 & 7 & 5 \\
1-2 años & 149 & 21,0 & 6 & 13 \\
2-3 años & 81 & 11,3 & 5 & 9 \\
3-4 años & 37 & 5,2 & 1 & 14 \\
4-5 años & 37 & 5,2 & 1 & 7 \\
$>5$ años & 108 & 15,1 & 8 & 37 \\
Total & 715 & 100 & 28 & 85 \\
\hline
\end{tabular}


yor frecuencia se siguen en más de dos servicios. El tiempo trascurrido desde la RT es de dos años o menos en el $58 \%$ de las visitas, en el $23 \%$ entre dos y cinco años y en el $21 \%$ a más de cinco (Tabla IV). El tiempo medio es de 2,5 años y la mediana de 1,5. El tiempo máximo transcurrido desde la radioterapia es de 18 años (Tabla II).

Se han detectado 28 sospechas de recidiva o metástasis ( $4 \%$ de visitas y $5,4 \%$ de pacientes) de las que se confirman 6 (21\% de sospecha, 1,2\% de pacientes): 4 locales y dos a distancia. A 85 pacientes se les ofrece el alta de los que 79 la aceptan $(11 \%$ de visitas y $15 \%$ de pacientes).

\section{Discusión}

Las características de esta serie (pacientes y localizaciones tumorales), así como del hospital y la organización asistencial pueden resultar bastante similar a lo que ocurre en otros escenarios de nuestro entorno. Resulta bien asumido que el mayor beneficio para el paciento oncológico se consigue con un abordaje multidisciplinario. Gracias a ello han mejorado los resultados finales en términos de supervivencia, calidad de vida y han aumentado también el número de tratamientos conservadores $s^{4,5}$. Para conseguirlo, durante la pasada década, los servicios clínicos han incorporado dos instrumentos muy importantes, como son los protocolos asistenciales consensuados y los comités de tumores multidisciplinarios ${ }^{5}$. Ambos instrumentos son especialmente necesarios en hospitales de ámbito general (no monográficos) en los que la atención a estos pacientes tiende a dispersarse entre muchos especialistas distintos.

La cantidad de trabajo que cualquier servicio de oncología dedica al seguimiento de los pacientes es muy elevado y la sobrecarga que produce suele reforzar la idea de que falta tiempo y espacio tranquilo para hablar con el enfermo. Su rendimiento puede resultar por todo ello cuestionable. En esta serie, acuden diariamente 20 pacientes y solo 1 ○ 2 se benefician de forma directa de este trabajo. La rentabilidad medida como sospechas/confirmación de recaídas es baja $(5,4 \%$ de pacientes), aún sin entrar a considerar el grado en que éstas pudieran o no ser susceptibles de tratamiento. Este comportamiento general es independiente del tiempo transcurrido desde que finalizó el tratamiento con radiaciones pudiendo hablarse de cierta "apropiación" de pacientes que choca con el consenso lógico desde el punto de vista del enfermo: el seguimiento debe ser el mínimo imprescindible, esto es, incluir el menor número de exploraciones, visitas y técnicas po- sible y basado en las mejores evidencias disponibles a cerca de su beneficio para el enfermo. Para mejorar esta situación han de darse dos circunstancias.

Tiene que reunir acuerdos en los protocolos más útiles y también acuerdo de quién y dónde es necesario llevarlo a cabo. El primer paso (la protocolización) es relativamente fácil de conseguir, mientras que el segundo implica cambios organizativos que parecen más dificultosos (las unidades funcionales o unidades clínicas, etc.) pero que vienen produciéndose empujados por los propios enfermos y por la necesidad de encontrar formas más eficientes de trabajar. Estos modelos incluyen además una única historia clínica y un único registro de eventos evolutivos. En ausencia de estos cambios, resulta evidente la necesidad de cambiar esta forma de trabajo y repartir el seguimiento de estos enfermos atendiendo a criterios que pueden y deben ser acordados. Tales podrían ser por localizaciones tumorales, por mayor responsabilidad en la carga de tratamiento, mayor influencia en caso de recidiva y su tratamiento o también en función del tiempo transcurrido. En los servicios de referencia como los de radioterapia un criterio vendría impuesto por el lugar de residencia de los enfermos.

\author{
Correspondencia: \\ Dr. J. Expósito Hernández \\ Servicio de Radioterapia \\ H. U. Virgen de las Nieves \\ Avda. de las Fuerzas Armadas, s/n \\ E-18011 Granada \\ E-mail: Jose.exposito.sspa@juntadeandalucia.es
}

\section{Bibliografía}

1. Wooldridge JE, Shahab N, Doll DG (guest editors). Introduction: Post-treatment Surveillance for potentially Curable Malignacies. Seminars in Oncology 2003; 30:337.

2. Miedma B, MacDonald I, Tatemichi J. Cancer follow-up care. Can Fam Phisician 2003; 49:890-5.

3. Colice GL, Rubins J, Unger M. Follow-up and Surveillance of the lung Cancer Patients Following curative-intent Therapy. Lung Cancer guidelines. CHEST 2003; 123: 272S83S.

4. Jeffery GM, Hickey BE, Hider P. Follow-up strategies for patients treated for non-metastatic colorectal cancer. The Cochrane Library, Issue 1, 2004. Chichester, UK: John Wiley \& Sons, Ltd.

5. American Society of Clinical Oncology Guidelines. Hitp://www.ASCO.org/ASCO990623html. 